\title{
A NEW CLASS OF ANALYTIC FUNCTIONS DEFINED BY CONVOLUTION WITH VARYING ARGUMENT
}

\author{
SHU-HAI LI, HUO TANG AND JING-YU YANG
}

\begin{abstract}
In this paper, we introduce a new class of analytic functions defined by convolution with varying argument and provide convolution properties and integral means inequalities for functions belonging to this new class. Some of our results generalize previously known results.
\end{abstract}

\section{Introduction}

Let $\mathscr{A}$ denote the class of functions of the form:

$$
f(z)=z+\sum_{j=2}^{\infty} a_{j} z^{j},
$$

which are analytic in the open unit disc $U=\{z \in C:|z|<1\}$. Also, by $T_{\gamma}(\gamma \in R)$ we denote the class of functions $f(z) \in \mathscr{A}$ of the form (1.1) for which all of non-vanishing coefficients satisfy the condition

$$
\arg \left(a_{n}\right)=\pi+(1-n) \gamma(n=2,3, \ldots) .
$$

For $\gamma=0$ we obtain the class $T_{0}$ of functions with negative coefficients. Moreover, we define

$$
T=\bigcup_{\gamma \in R} T_{\gamma}
$$

The class $T$ was introduced by Silverman [1] (see also [2], [3] and [18]). It is called the class of functions with varying argument of coefficients.

If $f$ of the form (1.1) and $g(z)=z+b_{2} z^{2}+\cdots+b_{j} z^{j}+\cdots$ are two functions in $\mathscr{A}$, then the convolution of $f$ and $g$ is denoted by $f * g$ and is given by

$$
(f * g)(z)=z+\sum_{j=2}^{\infty} a_{j} b_{j} z^{j}
$$

Corresponding author: Shu-Hai Li.

2010 Mathematics Subject Classification. 30C45, 30C50, $26 \mathrm{D} 15$.

Key words and phrases. Analytic functions, varying argument, subordination, convolution, integral means inequalities. 
For two functions $f$ and $g$ analytic in $U$, we say that the function $f$ is subordinate to $g$ in $U$, and write

$$
f(z) \prec g(z)(z \in U) .
$$

If there exists a Schwarz function $\omega$, which (by definition) is analytic in $U, \omega(0)=0$ and $|\omega(z)|<$ $1(z \in U)$, such that $f(z)=g(\omega(z))(z \in U)$. Indeed it is known that

$$
f(z) \prec g(z)(z \in U) \Leftrightarrow f(0)=g(0) \text { and } f(U) \subset g(U) .
$$

Furthermore, if the function $g$ is univalent in $U$, then we have the following equivalence $[4$, p.4]:

$$
f(z) \prec g(z)(z \in U) \Leftrightarrow f(0)=g(0) \text { and } f(U) \subset g(U) .
$$

Definition 1. A sequence $\left\{b_{j}\right\}_{j=1}^{\infty}$ of complex numbers is said to be a subordinating factor sequence if whenever $f(z)$ of the form (1.1) is analytic, univalent and convex in $U$, we have the subordination given by

$$
\sum_{j=1}^{\infty} a_{j} b_{j} z^{j} \prec f(z)\left(z \in U ; a_{1}=1\right)
$$

Now we define the following new class of analytic functions, and obtain some interesting results. Let $U(\phi, \psi ; \alpha, A, B)$ denote the subclass of $\mathscr{A}$ consisting of function $f(z)$ which satisfy the following inequality:

$$
\frac{f(z) * \phi(z)}{f(z) * \psi(z)}-\alpha\left|\frac{f(z) * \phi(z)}{f(z) * \psi(z)}-1\right|<\frac{1+A z}{1+B z}(\alpha \geq 0,-1 \leq B<A \leq 1),
$$

where

$$
\phi(z)=z+\sum_{j=2}^{\infty} \mu_{j} z^{j} \text { and } \psi(z)=z+\sum_{j=2}^{\infty} \eta_{j} z^{j}
$$

are analytic in $U$ such that $f(z) * \psi(z) \neq 0, \mu_{j}>\eta_{j} \geq 0(j \geq 2)$.

For suitable choices of $\phi, \psi$ and by specializing the parameters $\alpha, A, B$ involved in the class $U(\phi, \psi ; \alpha, A, B)$, we also obtain the following subclasses which were studied in many earlier works:

(1) $U\left(\frac{z}{(1-z)^{2}}, \frac{z}{1-z} ; 0, A, B\right)=S^{*}(A, B)$ and $U\left(\frac{z+z^{2}}{(1-z)^{3}}, \frac{z}{(1-z)^{2}} ; 0, A, B\right)=K(A, B)$

(Janowski. W [5] and K. S. Padmanabhan et al. [6]). For example, we have

$S^{*}(1,-1)=S^{*}$ (starlike functions $) ; K(1,-1)=K$ (convex functions);

(2) $U\left(\frac{z}{(1-z)^{2}}, \frac{z}{1-z} ; \alpha, 1,-1\right)=U S(\alpha)$ and $U\left(\frac{z+z^{2}}{(1-z)^{3}}, \frac{z}{(1-z)^{2}} ; \alpha, 1,-1\right)=U K(\alpha)$

(A. W. Goodman [7], W. C. Ma, D. Minda [8] and F. Ronning [9]);

(3) $U\left(\frac{z}{(1-z)^{2}}, \frac{z}{1-z} ; \alpha, 1-2 \beta,-1\right)=U S(\alpha, \beta)$ and $U\left(\frac{z+z^{2}}{(1-z)^{3}}, \frac{z}{(1-z)^{2}} ; \alpha, 1-2 \beta,-1\right)=U K(\alpha, \beta)$ (S. S. Hams et al. [10] and S. Shams et al.[11]); 
(4) $U\left(z+\sum_{j=2}^{\infty} j^{n+1} z^{j}, z+\sum_{j=2}^{\infty} j^{n} z^{j} ; \alpha, 1-2 \beta,-1\right)=U S_{n}(\alpha, \beta)$

(S.Shams et al. [13], R. Bharati et al. [14] and Wei-Ping Kuang, et al. [15]);

(5) $U\left(z+\sum_{j=2}^{\infty} j^{m} z^{j}, z+\sum_{j=2}^{\infty} j^{n} z^{j} ; \alpha, 1-2 \beta,-1\right)=U S_{m, n}(\alpha, \beta)$

$(0 \leq \alpha, 0 \leq \beta<1) \quad$ (S. S. Eker and S. Owa [16]);

(6) $U\left(z+\sum_{j=2}^{\infty} j^{m} z^{j}, z+\sum_{j=2}^{\infty} j^{n} z^{j} ; \alpha, 1-2 \beta,-1\right)=U S_{m, n}(\alpha, \beta)$

$(0 \leq \alpha, 0 \leq \beta<1) \quad$ (H.M. Srivastava and S.S. Eker [17]);

(7) $U(\phi, \psi ; 0, A, B)=U(\phi, \psi ; A, B) \quad$ (J.Dziok [18]) ;

(8) (6) $U\left(z+\sum_{j=2}^{\infty} j^{m} z^{j}, z+\sum_{j=2}^{\infty} j^{n} z^{j} ; \alpha, A, B\right)=U S_{m, n}(\alpha, A, B)$

$(0 \leq \alpha, 0 \leq \beta<1)$ (Shu-Hai Li and Huo Tang [19]).

Now, we define the classes of functions with varying argument of coefficients related to the class $U(\phi, \psi ; \alpha, A, B)$. Let us denote

$$
\begin{gathered}
T U_{\gamma}(\phi, \psi ; \alpha, A, B)=T_{\gamma} \cap U(\phi, \psi ; \alpha, A, B), \\
T U(\phi, \psi ; \alpha, A, B)=T \cap U(\phi, \psi ; \alpha, A, B) .
\end{gathered}
$$

In this paper, we establish a theorem concerning the subordination results of functions in the class $T U_{\gamma}(\phi, \psi ; \alpha, A, B)$.

\section{Main results}

To prove our main results, we need the following lemmas.

Lemma 1 ([20]). The sequence $\left\{b_{j}\right\}_{j=1}^{\infty}$ is a subordinating factor sequence if and only if

$$
\operatorname{Re}\left\{1+2 \sum_{j=1}^{\infty} b_{j} z^{j}\right\}>0,(z \in U) .
$$

Now, we prove the following lemma which gives a sufficient condition for functions belonging to the class $T U_{\gamma}(\phi, \psi ; \alpha, A, B)$.

Lemma 2. If $f(z) \in \mathscr{A}$ satisfies

$$
\sum_{j=2}^{\infty} \phi_{j}\left(\mu_{j}, \eta_{j}, \alpha, A, B\right)\left|a_{j}\right| \leq A-B
$$

where

$$
\phi_{j}\left(\mu_{j}, \eta_{j}, \alpha, A, B\right)=(1+(1+|B|) \alpha)\left(\mu_{j}-\eta_{j}\right)+\left|B \mu_{j}-A \eta_{j}\right|
$$


For some $\alpha \geq 0,-1 \leq B<A \leq 1, \mu_{j}>\eta_{j} \geq 0(j \geq 2)$, then $f(z) \in T U_{\gamma}(\phi, \psi ; \alpha, A, B)$.

Proof. Suppose that (2.1) is true for $\alpha \geq 0,-1 \leq B<A \leq 1$. For $f(z) \in \mathscr{A}$, let us define the function $p(z)$ by

$$
p(z)=\frac{f(z) * \phi(z)}{f(z) * \psi(z)}-\alpha\left|\frac{f(z) * \phi(z)}{f(z) * \psi(z)}-1\right| .
$$

It suffices to show that

$$
\left|\frac{p(z)-1}{A-B p(z)}\right|<1(z \in U)
$$

We note that

$$
\begin{aligned}
& \left|\frac{p(z)-1}{A-B p(z)}\right| \\
& =\left|\frac{f(z) * \phi(z)-\alpha e^{i \theta}|f(z) * \phi(z)-f(z) * \psi(z)|-f(z) * \psi(z)}{A f(z) * \psi(z)-B\left(f(z) * \phi(z)-\alpha e^{i \theta}|f(z) * \phi(z)-f(z) * \psi(z)|\right)}\right| \\
& =\left|\frac{(f(z) * \phi(z)-f(z) * \psi(z))-\alpha e^{i \theta}|f(z) * \phi(z)-f(z) * \psi(z)|}{(A-B) f(z) * \psi(z)-B\left((f(z) * \phi(z)-f(z) * \psi(z))-\alpha e^{i \theta}|f(z) * \phi(z)-f(z) * \psi(z)|\right)}\right| \\
& =\left|\frac{\sum_{j=2}^{\infty}\left(\mu_{j}-\eta_{j}\right) a_{j} z^{j-1}-\alpha e^{i \theta}\left|\sum_{j=2}^{\infty}\left(\mu_{j}-\eta_{j}\right) a_{j} z^{j-1}\right|}{(A-B)-\sum_{j=2}^{\infty}\left(B \mu_{j}-A \eta_{j}\right) a_{j} z^{j-1}-\alpha B e^{i \theta}\left|\sum_{j=2}^{\infty}\left(\mu_{j}-\eta_{j}\right) a_{j} z^{j-1}\right|}\right| \\
& \leq \frac{\sum_{j=2}^{\infty}\left|\mu_{j}-\eta_{j}\right|\left|a_{j}\right||z|^{j-1}+\alpha\left|e^{i \theta}\right| \sum_{j=2}^{\infty}\left|\mu_{j}-\eta_{j}\right|\left|a_{j}\right||z|^{j-1}}{(A-B)-\sum_{j=2}^{\infty}\left|B \mu_{j}-A \eta_{j}\right|\left|a_{j}\right||z|^{j-1}-\alpha|B|\left|e^{i \theta}\right| \sum_{j=2}^{\infty}\left|\mu_{j}-\eta_{j}\left\|a_{j}\right\| z\right|^{j-1}} \\
& \leq \frac{\sum_{j=2}^{\infty}\left|\mu_{j}-\eta_{j}\right|\left|a_{j}\right|+\alpha \sum_{j=2}^{\infty}\left|\mu_{j}-\eta_{j}\right|\left|a_{j}\right|}{(A-B)-\sum_{j=2}^{\infty}\left|B \mu_{j}-A \eta_{j}\right|\left|a_{j}\right|-\alpha \sum_{j=2}^{\infty}\left|\mu_{j}-\eta_{j}\right|\left|a_{j}\right|}
\end{aligned}
$$

The last expression is bounded above by 1 , if

$$
\sum_{j=2}^{\infty}\left|\mu_{j}-\eta_{j}\right|\left|a_{j}\right|+\alpha \sum_{j=2}^{\infty}\left|\mu_{j}-\eta_{j}\right|\left|a_{j}\right| \leq(A-B)-\sum_{j=2}^{\infty}\left|B \mu_{j}-A \eta_{j}\right|\left|a_{j}\right|-\alpha \sum_{j=2}^{\infty}\left|\mu_{j}-\eta_{j}\right|\left|a_{j}\right|
$$

which is equivalent to our condition (2.1). This completes the proof of our theorem.

Remark. Taking $\phi(z)=z+\sum_{j=2}^{\infty} j^{m} z^{j}, \psi(z)=z+\sum_{j=2}^{\infty} j^{n} z^{j}(0 \leq \alpha, 0 \leq \beta<1)$, we obtain the improved result of Theorem 1 in the paper [19].

Lemma 3. Let $f(z)$ be a function of the form (1.1), with (1.2). Then $f(z) \in T U_{\gamma}(\phi, \psi ; \alpha, A, B)$ if and only if the inequality (2.1) holds true.

Proof. In view of Lemma 2, we need only show that each function $f(z)$ from the class $T U_{\gamma}(\phi, \psi$; $\alpha, A, B)$ satisfies the coefficient inequality (2.1). Let $f(z) \in T U_{\gamma}(\phi, \psi ; \alpha, A, B)$, by (2.3) and (1.1), we obtain

$$
\left|\frac{\sum_{j=2}^{\infty}\left(\mu_{j}-\eta_{j}\right) a_{j} z^{j-1}-\alpha e^{i \theta}\left|\sum_{j=2}^{\infty}\left(\mu_{j}-\eta_{j}\right) a_{j} z^{j-1}\right|}{(A-B)-\sum_{j=2}^{\infty}\left(B \mu_{j}-A \eta_{j}\right) a_{j} z^{j-1}-\alpha B e^{i \theta}\left|\sum_{j=2}^{\infty}\left(\mu_{j}-\eta_{j}\right) a_{j} z^{j-1}\right|}\right|<1 \quad(z \in U) .
$$


Therefore, putting $z=r e^{i \gamma}(0 \leq \gamma<1)$, and applying (1.2) we have

$$
\frac{\sum_{j=2}^{\infty}\left|\mu_{j}-\eta_{j}\right|\left|a_{j}\right| r^{j-1}+\alpha \sum_{j=2}^{\infty}\left|\mu_{j}-\eta_{j}\right|\left|a_{j}\right| r^{j-1}}{(A-B)-\sum_{j=2}^{\infty}\left|B \mu_{j}-A \eta_{j}\right|\left|a_{j}\right| r^{j-1}-\alpha \sum_{j=2}^{\infty}\left|\mu_{j}-\eta_{j} \| a_{j}\right| r^{j-1}}<1 .
$$

It is clear that the denominator of the left hand said cannot vanish for $0 \leq r<1$, thus, we obtain

$$
\sum_{j=2}^{\infty}\left((1+(1+|B|) \alpha)\left(\mu_{j}-\eta_{j}\right)+\left|B \mu_{j}-A \eta_{j}\right|\right)\left|a_{j}\right| r^{j-1},
$$

which, upon letting $r \rightarrow 1^{-}$, readily yields the assertion (2.1).

Since the condition (2.1) is independent of $\gamma$, Lemma 2 yields the following theorem.

From Lemma 3, we have the following Theorem 1 and Theorem 2.

Theorem 1. If a function of $f(z)$ the form (1.1), $f(z) \in T U_{\gamma}(\phi, \psi ; \alpha, A, B)$, then

$$
\left|a_{j}\right| \leq \frac{A-B}{\phi_{j}\left(\mu_{j}, \eta_{j}, \alpha, A, B\right)}(j=2,3, \ldots),
$$

where $\phi_{j}\left(\mu_{j}, \eta_{j}, \alpha, A, B\right)$ is defined by (2.2), the result is sharp. The functions $f_{j, \gamma}(z)$ of the form

$$
f_{j, \gamma}(z)=z-\frac{A-B}{\phi_{j}\left(\mu_{j}, \eta_{j}, \alpha, A, B\right)} e^{i(1-j) \gamma} z^{j}(z \in U ; j=2,3, \ldots)
$$

are the extreme functions.

Theorem 2. Let a function $f(z)$ of the form (1.1) belong to class $T U_{\gamma}(\phi, \psi ; \alpha, A, B)$. If the sequence $\left\{\phi_{j}\left(\mu_{j}, \eta_{j}, \alpha, A, B\right)\right\}_{j=1}^{\infty}$ defined by (2.2) satisfies the inequality

$$
\phi_{2}\left(\mu_{2}, \eta_{2}, \alpha, A, B\right) \leq \phi_{j}\left(\mu_{j}, \eta_{j}, \alpha, A, B\right)(j=2,3, \ldots),
$$

then

$$
\sum_{j=2}^{\infty} a_{j} \leq \frac{2(A-B)}{\phi_{2}\left(\mu_{2}, \eta_{2}, \alpha, A, B\right)}
$$

Moreover, if

$$
j \phi_{2}\left(\mu_{2}, \eta_{2}, \alpha, A, B\right) \leq 2 \phi_{j}\left(\mu_{j}, \eta_{j}, \alpha, A, B\right)(j=2,3, \ldots),
$$

then

$$
\sum_{j=2}^{\infty} j a_{j} \leq \frac{2(A-B)}{\phi_{2}\left(\mu_{2}, \eta_{2}, \alpha, A, B\right)}
$$

Employing the technique used earlier by Attiya [21], Srivastava and Attiya [22] and M. K. Aouf [23], we prove: 
Theorem 3. Let $f(z) \in T U_{\gamma}(\phi, \psi ; \alpha, A, B)$. Then

$$
\frac{\phi_{2}\left(\mu_{2}, \eta_{2}, \alpha, A, B\right)}{2\left(A-B+\phi_{2}\left(\mu_{2}, \eta_{2}, \alpha, A, B\right)\right)}(f * g)(z) \prec g(z)
$$

for every function $g$ in $K$, and

$$
\operatorname{Re} f(z)>-\frac{1}{2\left(A-B+\phi_{2}\left(\mu_{2}, \eta_{2}, \alpha, A, B\right)\right)}, \quad(z \in U) .
$$

The constant factor $\frac{\phi_{2}\left(\mu_{2}, \eta_{2}, \alpha, A, B\right)}{2\left(A-B+\phi_{2}\left(\mu_{2}, \eta_{2}, \alpha, A, B\right)\right)}$ cannot be replaced by a larger number.

Proof. Let $f(z) \in T U_{\gamma}(\phi, \psi ; \alpha, A, B)$ and suppose that $g(z)=z+\sum_{j=2}^{\infty} c_{j} z^{j} \in K$. Then

$$
\frac{\phi_{2}\left(\mu_{2}, \eta_{2}, \alpha, A, B\right)}{2\left(A-B+\phi_{2}\left(\mu_{2}, \eta_{2}, \alpha, A, B\right)\right)}(f * g)(z)=\frac{\phi_{2}\left(\mu_{2}, \eta_{2}, \alpha, A, B\right)}{2\left(A-B+\phi_{2}\left(\mu_{2}, \eta_{2}, \alpha, A, B\right)\right)}\left(z+\sum_{j=2}^{\infty} a_{j} c_{j} z^{j}\right)
$$

Thus, by Definition 1 the subordination result (2.7) will hold true if the sequence

$$
\left\{\frac{\phi_{2}\left(\mu_{2}, \eta_{2}, \alpha, A, B\right)}{2\left(A-B+\phi_{2}\left(\mu_{2}, \eta_{2}, \alpha, A, B\right)\right)} a_{j}\right\}_{j=1}^{\infty}
$$

is a subordinating factor sequence, with $a_{1}=1$. In view of Lemma 1 , this is equivalent to the following inequality:

$$
\operatorname{Re}\left\{1+2 \sum_{j=1}^{\infty} \frac{a_{j} z^{j}}{2\left(A-B+\phi_{2}\left(\mu_{2}, \eta_{2}, \alpha, A, B\right)\right)}\right\}, \quad(z \in U) .
$$

Thus, by (2.5) for $|z|=r<1$, we have

$$
\begin{aligned}
\operatorname{Re} & \left\{1+2 \sum_{j=1}^{\infty} \frac{a_{j} z^{j}}{2\left(A-B+\phi_{2}\left(\mu_{2}, \eta_{2}, \alpha, A, B\right)\right)}\right\} \\
& =\operatorname{Re}\left\{1+\frac{z}{\left(A-B+\phi_{2}\left(\mu_{2}, \eta_{2}, \alpha, A, B\right)\right)}+\sum_{j=2}^{\infty} \frac{a_{j} z^{j}}{\left(A-B+\phi_{2}\left(\mu_{2}, \eta_{2}, \alpha, A, B\right)\right)}\right\} \\
& \geq 1-\frac{r}{\left(A-B+\phi_{2}\left(\mu_{2}, \eta_{2}, \alpha, A, B\right)\right)}-\frac{1}{\left(A-B+\phi_{2}\left(\mu_{2}, \eta_{2}, \alpha, A, B\right)\right)} \sum_{j=2}^{\infty} \phi_{j}\left(\mu_{j}, \eta_{j}, \alpha, A, B\right)\left|a_{j}\right| r^{j} \\
& >1-\frac{\phi_{2}\left(\mu_{2}, \eta_{2}, \alpha, A, B\right)}{\left(A-B+\phi_{2}\left(\mu_{2}, \eta_{2}, \alpha, A, B\right)\right)} r-\frac{A-B}{\left(A-B+\phi_{2}\left(\mu_{2}, \eta_{2}, \alpha, A, B\right)\right)} r>0
\end{aligned}
$$

where we have also made use of assertion (2.1) of Lemma 2. Thus (2.9) holds true in $U$. This proves the inequality (2.7). The inequality (2.8) follows from (2.7) by taking the convex function

$$
g(z)=\frac{z}{1-z}=z+\sum_{j=2}^{\infty} z^{j}
$$


Next we observe that the function $f_{2, \gamma}(z)$ of the form (2.4) belongs to the class $T U_{\gamma}(\phi, \psi$; $\alpha, A, B)$.

It is easily verified that

$$
\min \left\{\operatorname{Re}\left(\frac{\phi_{2}\left(\mu_{2}, \eta_{2}, \alpha, A, B\right)}{2\left(A-B+\phi_{2}\left(\mu_{2}, \eta_{2}, \alpha, A, B\right)\right)} f_{2, \gamma}(z)\right)\right\}=-\frac{1}{2}(z \in U) .
$$

This shows that the constant $\frac{\phi_{2}\left(\mu_{2}, \eta_{2}, \alpha, A, B\right)}{2\left(A-B+\phi_{2}\left(\mu_{2}, \eta_{2}, \alpha, A, B\right)\right)}$ cannot be replaced by any larger one.

Corollary 1. Let $f(z) \in T U(\phi, \psi ; \alpha, A, B)$. Then

$$
\frac{\phi_{2}\left(\mu_{2}, \eta_{2}, \alpha, A, B\right)}{2\left(A-B+\phi_{2}\left(\mu_{2}, \eta_{2}, \alpha, A, B\right)\right)}(f * g)(z) \prec g(z)
$$

for every function $g$ in $K$, and

$$
\operatorname{Re} f(z)>-\frac{1}{2\left(A-B+\phi_{2}\left(\mu_{2}, \eta_{2}, \alpha, A, B\right)\right)}, \quad(z \in U) .
$$

The constant factor $\frac{\phi_{2}\left(\mu_{2}, \eta_{2}, \alpha, A, B\right)}{2\left(A-B+\phi_{2}\left(\mu_{2}, \eta_{2}, \alpha, A, B\right)\right)}$ cannot be replaced by a larger number.

Now, we discuss the integral means inequalities of functions $f(z)$ in $T U_{\gamma}(\phi, \psi ; \alpha, A, B)$.

Lemma 4 (Littlewood [24]). If $f(z)$ and $g(z)$ are analytic in $U$ with $f(z)<g(z)$, then for $\mu>0$ and $z=r e^{i \theta}(0<r<1)$

$$
\int_{0}^{2 \pi}|f(z)|^{\mu} d \theta \leq \int_{0}^{2 \pi}|g(z)|^{\mu} d \theta .
$$

Theorem 4. Let $f(z)$ in $T U_{\gamma}(\phi, \psi ; \alpha, A, B)$ and suppose that $f_{n}(z)$ is defined by (2.4). If there exists an analytic function $\omega(z)$ given by

$$
\omega(z)=\frac{\phi_{n}\left(\mu_{n}, \eta_{n}, \alpha, A, B\right)}{(A-B) e^{i(1-n) \gamma}} \sum_{j=2}^{\infty} a_{j} z^{j-1} \quad(n=2,3, \ldots)
$$

then for $z=r e^{i \theta}(0<r<1)$ and $\mu>0$,

$$
\int_{0}^{2 \pi}\left|f\left(r e^{i \theta}\right)\right|^{\mu} d \theta \leq \int_{0}^{2 \pi}\left|f_{n}\left(r e^{i \theta}\right)\right|^{\mu} d \theta
$$

Proof. For $z=r e^{i \theta}(0<r<1)$, we must show that

$$
\int_{0}^{2 \pi}\left|1+\sum_{j=2}^{\infty} a_{j} z^{j-1}\right|^{\mu} d \theta \leq \int_{0}^{2 \pi}\left|1+\frac{(A-B) e^{i(1-n) \gamma}}{\phi_{n}\left(\mu_{n}, \eta_{n}, \alpha, A, B\right)} z^{n-1}\right|^{\mu} d \theta(\mu>0) .
$$

Thus by applying Littlewood's subordination theorem, it would suffice to show that

$$
1+\sum_{j=2}^{\infty} a_{j} z^{j-1} \prec 1+\frac{(A-B) e^{i(1-n) \gamma}}{\phi_{n}\left(\mu_{n}, \eta_{n}, \alpha, A, B\right)} z^{n-1} .
$$


By setting

$$
1+\sum_{j=2}^{\infty} a_{j} z^{j-1}=1+\frac{(A-B) e^{i(1-n) \gamma}}{\phi_{n}\left(\mu_{n}, \eta_{n}, \alpha, A, B\right)} \omega(z)
$$

we find that

$$
\omega(z)=\frac{\phi_{n}\left(\mu_{n}, \eta_{n}, \alpha, A, B\right)}{(A-B) e^{i(1-n) \gamma}} \sum_{j=2}^{\infty} a_{j} z^{j-1},
$$

which readily yields $\omega(0)=0$.

Therefore, we have

$$
|\omega(z)|=\left|\frac{\phi_{n}\left(\mu_{n}, \eta_{n}, \alpha, A, B\right)}{(A-B) e^{i(1-n) \gamma}} \sum_{j=2}^{\infty} a_{j} z^{j-1}\right| \leq \frac{\phi_{n}\left(\mu_{n}, \eta_{n}, \alpha, A, B\right)}{A-B} \sum_{j=2}^{\infty}\left|a_{j}\right||z|^{j-1} \leq|z|<1,
$$

by means of hypothesis and Lemma 4.

We remark in conclusion that, by suitably specializing the parameters involved in the results presented in this paper, we can deduce numerous further corollaries and consequences of each of these results.

\section{Acknowledgements}

The present investigation was partly supported by the Natural Science Foundation of Inner Mongolia under Grant 2009MS0113.

\section{References}

[1] H. Silverman, Univalent functions with varying arguments, Houston J. Math., 7(1981), 283-287.

[2] H. M. Srivastava, S. Owa, Certain classes of analytic functions with varying arguments, J. Math. Anal. Appl., 136(1988), 217-228.

[3] H. Silverman, G. Murugusundaramoorthy and K. Vijaya, A class of functions defined by Dziok-Srivastava operator, Kyungpook Math. J., 49(2009), 95-106.

[4] S. S. Miller and P. T. Mocanu, Differential Subordinations: Theory and Applications, in: Series on Monographs and Textbooks in Pure and Appl. Math., vol. 255, Marcel Dekker, Inc, New York, 2000.

[5] W. Janowski, Some extreme problems for certain families of analytic functions, Ann. Polon. Math., 28(1973), 648-658.

[6] K. S. Padmanabhan and M. S. Ganesan, Convolutions of certain classes of univalent functions with negative coefficients, Indian J. Pure Appl. Math., 19(1988), 880-889.

[7] A. W. Goodman, On uniformly starlike functions, J. Math. Anal. Appl., 155(1991), 364-370.

[8] W. C. Ma and D. Minda, Uniformly convex functions, Ann. Polon. Math., 57(1992), 165-175.

[9] F. Ronning, Uniformly convex functions and a corresponding class of starlike functions, Proc. Amer. Math. Soc., 118(1993), 189-196.

[10] S. S. Hams, S. R. Kulkarni and J. M. Jahangiri, Classes of uniformly starlike and convex functions, Internat. J. Math. Math. Sci., 55 (2004), 2959-2961.

[11] S. Shams and S. R. Kulkarni, On a class of univalent functions defined by Ruscheweyh derivatives, KYUNGPOOK Math. J., 43(2003), 579-585. 
[12] G. S. Salagean, Subclasses of univalent functions, in: Complex Analysis: Fifth Romanian-Finnish Seminar, Part 1 (Bucharest, 1981), in: Lecture. Notes in Mathematics, vol. 1013, Springer-Verlag, Berlin, Heidelberg, New York, 1983, pp. 362-372.

[13] S. Shams, S. R. Kulkarni and J. M. Jahangiri, Classes of uniformly starlike and convex functions, Internat. J. Math. Math. Sci., 2004(2004), Issue 55, 2959-2961.

[14] R. Bharati, R. Parvatham and A. Swaminathan, On subclasses of uniformly convex functions and corresponding class of starlike functions, Tamkang J. Math., 28 (1997), 17-32.

[15] W.-P. Kuang, Y. Sun, Z.-G. Wang, On Quasi-Hadamard product of certain classes of analytic functions, Bulletin of Mathematical Analysis and Applications, 2(2009), 36-46.

[16] S. S. Eker, S. Owa, Certain classes of analytic functions involved Salagean operator, J. Inequal. Pure Appl. Math., 10 (2009), 12-22.

[17] H. M. Srivastava, S. S. Eker, Some applications of a subordination theorem for a class of analytic functions, Appl. Math. Lett., 21(2008), 394-399.

[18] J. Dziok, Certain inequalities for classes of analytic functions with varying argument of coefficients, Mathematical Inequalities and Applications, 2(2011), 389-398.

[19] S.-H. Li, H. Tang, Certain new classes of analytic functions defined by using the salagean operator, Bulletin of Mathematical Analysis and Applications, 4 (2010), 62-75.

[20] H. S. Wilf, Subordinating factor sequence for convex maps of the unit circle, Proc. Amer. Math. Soc., 12(1961), 689-693.

[21] A. A. Attiya, On some application of a subordination theorems, J. Math. Anal. Appl., 311 (2005), $489-494$.

[22] H. M. Srivastava and A. A. Attiya, Some subordination results associated with certain subclass of analytic functions, J. Inequal. Pure Appl. Math., 5 (2004), 1-6.

[23] M. K. Aouf, Subordination properties for a certain class of analytic functions defined by the Salagean operator, Appl. Math. Lett., 22 (2009), 1581-1585.

[24] J. E. Littlewood, On inequalities in the theory of functions, Proc. London Math. Soc., 3(1925), 481-519.

School of Mathematics and Statistics, Chifeng University, Chifeng 024001, Inner Mongolia, China.

E-mail: lishms66@sina.com

School of Mathematics and Statistics, Chifeng University, Chifeng 024001, Inner Mongolia, China; School of Mathematical Sciences, Beijing Normal University, Beijing 100875, China.

E-mail: thth2009@tom.com

School of Mathematics and Statistics, Chifeng University, Chifeng 024001, Inner Mongolia, China; School of Mathematical Sciences, Dalian University of Technology, Dalian 116024, Liaoning, China.

E-mail: yjy923@163.com 